\title{
O Internato de Formação Especializada em Neurocirurgia: Qual o Atual Panorama Nacional?
}

\section{Neurosurgical Residency in Portugal: What is the National Panorama?}

\author{
Lídia NUNES DIAS ${ }^{1}$, Pedro PINTO LEITE², Carla REIZINHO ${ }^{1}$, José CABRAL ${ }^{1}$ \\ Acta Med Port 2022 Jan;35(1):20-29 - https://doi.org/10.20344/amp.15110
}

\section{RESUMO}

Introdução: Em Portugal, o número de médicos internos em Neurocirurgia tem vindo a aumentar progressivamente ao longo dos anos, contudo esta evolução não tem sido acompanhada de estudos que permitam compreender o estado atual da formação. Foi objetivo deste estudo caracterizar e quantificar a satisfação na formação especializada em Neurocirurgia, em Portugal, no ano de 2019. Material e Métodos: Estudo quantitativo, observacional e transversal baseado num questionário original enviado eletronicamente aos internos e recém-especialistas de Neurocirurgia entre outubro e dezembro de 2019. Incluiu-se perguntas sobre características e satisfação em termos de formação teórica, prática, entre outras.

Resultados: Obtiveram-se 37 respostas em médicos com cerca de $29,0( \pm 4,0)$ anos, $78,4 \%$ homens e $54,1 \%$ provenientes de centros do Centro/Sul/llhas. Do total de respostas obtidas, $51,4 \%$ vieram de internos dos três primeiros anos. Quanto à formação teórica, evidenciou-se insatisfação em relação às reuniões de morbimortalidade $(59,5 \%)$, existência de sessões/laboratório anatómico (89,2\%), participação no ensino graduado (64,9\%) e em investigação $(64,9 \%)$. Quanto à formação prática, a insatisfação evidencia-se apenas em relação à consulta externa $(56,8 \%)$. A primeira intervenção cirúrgica tende a ser realizada no primeiro mês de internato, no primeiro ano. Por ordem crescente, a primeira cirurgia é de trauma craniano (5,09 $\pm 4,59$ meses), patologia de liquor (5,95 $\pm 4,3$ meses), nervos periféricos (6,0 $\pm 7,0$ meses), craniotomia (6,59 $\pm 3,88$ meses) e patologia lombar (11,41 $\pm 1,5$ meses). A cirurgia pediátrica é a última a ser iniciada (19,36 $\pm 20,0$ meses). Parece existir satisfação geral com a avaliação anual $(59,5 \%)$ mas não com a final (37,8\%).

Conclusão: Este estudo cumpriu o objetivo principal de ser um ponto de partida na caracterização dos centros neurocirúrgicos portugueses e da satisfação no internato de formação especializada em Neurocirurgia.

Palavras-chave: Educação de Pós-Graduação em Medicina; Internato e Residência Neurocirurgia/educação; Portugal

\section{ABSTRACT}

Introduction: In Portugal, the number of neurosurgery residents has been rising steadily. However, there are no robust studies assesing the level of satisfaction and quality of the current training programs. The aim of this study was to describe and quantify the level of satisfaction about Neurosurgery residency in 2019, in Portugal.

Material and Methods: Quantitative observational cross-sectional study based on an original questionnaire about the level of satisfaction of neurosurgical training in Portugal in 2019, sent electronically to residents and young consultants between October and December 2019.

Results: A total of 37 responses were obtained from physicians aged around 29.0 ( \pm 4.0 ) years old, of which $78.4 \%$ were men and $54.1 \%$ from centers in the center/south of the country/islands. Overall, $51.4 \%$ of the answers came from first three years' residents. As for the theoretical training, there was dissatisfaction with the morbidity and mortality meetings (59.5\%), existence of sessions/anatomical lab (89.2\%), participation in medical education (64.9\%) and in research (64.9\%). As for practical training, there was dissatisfaction only towards outpatient clinics $(56.8 \%)$. There is a tendency for the first surgery to occur in the first month of residency and, in ascending order, firstly a cranial trauma surgery (5.09 \pm 4.59 months), then for cerebrospinal fluid diseases $(5.95 \pm 4.3$ months $)$, peripheral nerves (6.0 \pm 7.0 months), craniotomy ( $6.59 \pm 3.88$ months) and lumbar spine diseases (11.41 \pm 1.5 months). Pediatric surgery was the last type of surgery to begin $(19.36 \pm 20.0$ months). There seems to be a generalized satisfaction with the annual (59.5\%) but not with the final examination $(37.8 \%)$.

Conclusion: This study has succeed at being a better description of the Portuguese neurosurgical centers and of the level of satisfaction about neurosurgical training in Portugal.

Keywords: Education, Medical, Graduate; Internship and Residency; Neurosurgery/education; Portugal

\section{INTRODUÇÃO}

Em Portugal, o número de médicos internos e especialistas em Neurocirurgia tem aumentado progressivamente ao longo dos anos, à semelhança do que tem acontecido com outras especialidades, na tentativa de compensação da crescente formação pré-graduada, ainda que de forma ligeira e aparentemente controlada. ${ }^{1}$ Apesar do Ministério da Saúde procurar por vezes enquadrar os recursos médicos existentes com as necessidades populacionais, ${ }^{1}$ não existem estudos que visem caracterizar o internato médico de Neurocirurgia propriamente dito, a sua qualidade e a satisfação com o mesmo, à semelhança de outros países. ${ }^{2-5}$

\section{O internato de formação especializada em Portugal}

Segundo os últimos dados do Ministério da Saúde, desde 2014 criaram-se nove a 12 centros de formação especializada em Neurocirurgia, alguns deles com idoneidade parcial (cinco em 12), contudo nem sempre com possibilidade de vaga todos os anos. ${ }^{1}$ Cada centro tende a

1. Departamento de Neurocirurgia. Hospital Egas Moniz. Centro Hospitalar Lisboa Ocidental. Lisboa. Portugal.

2. Unidade de Saúde Pública. Agrupamento de Centros de Saúde Almada-Seixal. Almada. Portugal.

$\triangle$ Autor correspondente: Lídia Nunes Dias. Idias@chlo.min-saude.pt

Recebido: 17 de outubro de 2020 - Aceite: 23 de fevereiro de 2021 - Online issue published: 03 de janeiro de 2022

Copyright @ Ordem dos Médicos 2022 
receber entre um a dois internos, de acordo com o que é pedido pelo serviço/centro hospitalar, avaliado como capaz pelo Colégio de Especialidade de Neurocirurgia da Ordem dos Médicos e pelo Conselho Nacional do Internato Médico (CNIM), e por último decidido pelo Ministério da Saúde (ACSS - Administração Central do Sistema de Saúde, IP), de acordo com os recursos gerais do mesmo. Estima-se que existam anualmente cerca de 50 médicos internos de neurocirurgia em Portugal. ${ }^{1}$

Em 2019, o internato médico de Neurocirurgia seguia a Portaria número 146/98 de 9 de março, onde é referido um programa de formação com a duração de seis anos (72 meses). ${ }^{6}$ Esta legislação viria a ser revogada pela Portaria n 393/2019 de 6 de Novembro, não sendo aplicável na integra a quem respondeu a este inquérito. Durante estes anos, o médico interno tem objetivos de desempenho a nível cirúrgico (números mínimos de intervenções por grupo de patologia), objetivos de conhecimento (capacidade de diagnosticar e tratar) e de produção científica (comunicações e publicações). O programa termina com orientações para uma avaliação anual com duas componentes: desempenho (capacidade de execução técnica, interesse pela valorização profissional, responsabilidade profissional e relações humanas de trabalho) e conhecimento (contínua, complementada por uma prova de apreciação e discussão de um relatório anual de atividades produzido pelo médico interno).

O programa de formação é da responsabilidade do Colégio de Especialidade de Neurocirurgia da Ordem dos Médicos, que procura alinhá-lo com as normas europeias e, assim, garantir uma uniformização e melhoria contínua da qualidade assistencial. ${ }^{1}$

Para serem reconhecidos formalmente como centros de formação de médicos internos em Neurocirurgia, segundo o Colégio da Especialidade de Neurocirurgia, os serviços devem ter um número de casos e variedade de patologias suficiente para proporcionar uma formação adequada, um mínimo de quatro orientadores de formação, 30 camas no serviço e a 10 camas de Cuidados Intensivos por milhão de habitantes da área de influência direta. . $^{1,8}$ É ainda exigido que estes serviços estejam equipados com microscópio, apoio de neuro-navegação, aspirador ultrassónico, endoscópio, motores como craniótomo e brocas de desbaste ósseo, intensificador de imagem, possibilidade de utilização de monitorização eletrofisiológica intraoperatória e acesso a bloco de ambulatório. ${ }^{1,8}$ Outro requisito fundamental é a existência de urgência de 24 horas com capacidade de contribuir com determinados números (dados em tabela valores mínimos e ótimos) de cirurgias para o currículo ao interno no final da sua formação. ${ }^{1,8}$ Nestes serviços deverão ainda existir pelo menos duas salas cirúrgicas preparadas para Neurocirurgia, com anestesistas formados em neuroanestesia e disponíveis 24 horas por dia. ${ }^{1,8} \mathrm{O}$ programa de formação deve integrar um estágio de Neurocirurgia Pediátrica e um estágio de Neurocirurgia Funcional, realizados no local de formação do médico interno ou noutro local com idoneidade reconhecida pela Ordem dos Médicos. ${ }^{1,8}$
Apesar de todas estas recomendações, existe a perceção de que nem todos os serviços estão equipados de forma igual e de que existem eventuais assimetrias na formação do médico interno.

\section{Enquadramento e formulação do estudo}

Em Portugal, os estudos sobre a formação especializada em Neurocirurgia são escassos, impedindo a identificação das referidas dissemelhanças formativas existentes a nível nacional e internacional, nomeadamente quanto ao número de horas de trabalho, ao género dos médicos internos, à publicação de artigos e à carreira académica. ${ }^{10-18}$

É opinião dos autores que a satisfação dos médicos internos pode ser influenciada por vários fatores previamente identificados em estudos internacionais, como características e organização dos serviços, carga horária, distribuição de tarefas e oportunidades formativas (teóricas e práticas) disponibilizadas.

Em termos de constituição e organização dos serviços, acredita-se que esta satisfação poderá eventualmente variar de acordo com o número de internos em cada um, podendo ser maior no serviço de urgência (SU) (onde não se verificam tantos períodos de urgência), mas menor no bloco (por menos oportunidades). Um maior número de médicos com doutoramento poderá estar possivelmente associado a maior produção científica do serviço, o que poderá contribuir para uma maior satisfação dos médicos internos.

A realização de sessões clínicas ou journal clubs, eventualmente por permitirem uma revisão de temas importantes e atualização de conhecimento, poderá ser uma mais-valia e motivo de satisfação com em termos de formação teórica, assim como as reuniões multidisciplinares, que dependem da existência de determinadas subespecialidades num serviço, reuniões de morbilidade e mortalidade, sessões anatómicas e acesso a laboratório de anatomia. A colaboração no ensino graduado de Medicina, através de aulas ou do acompanhamento de teses de mestrado (ou outros documentos de produção científica como comunicações ou publicações) poderá estar associada a uma maior satisfação, eventualmente por permitir um contacto constante com informação atualizada. Esta satisfação também parece estar associada à realização de estudos de investigação no local de colocação, e à formação externa, incluindo cursos ou estágios nacionais e internacionais.

Quanto à formação prática, pensa-se que o desempenho e a satisfação dos médicos internos poderão estar associados a uma melhor organização do serviço, carga horária, distribuição de tarefas, ao apoio assistencial e ao número de doentes observados no serviço de Urgência, na enfermaria e nas consultas. Mais importante ainda, ao se tratar de uma especialidade cirúrgica, pensa-se que as oportunidades no bloco poderão ser uma das componentes mais relevantes e contribuidoras para a satisfação na formação prática e, quiçá, do Internato no geral.

Parece existir uma grande variabilidade entre países europeus quanto a aspetos práticos e teóricos da formação em Neurocirurgia, apesar da tentativa de 
harmonização dos programas nas últimas duas décadas através da criação de normas europeias. ${ }^{8,9}$ Segundo Stienen et al, os médicos internos portugueses são dos mais satisfeitos quanto à realização de journal clubs, ao início de prática cirúrgica precoce e ao contacto com um grande número de patologias. No entanto, esta satisfação encontra-se abaixo da média europeia quanto ao número de reuniões neurorradiológicas. ${ }^{9}$

Assim, a realização de um inquérito semelhante ao realizado ao nível europeu ${ }^{9}$ poderia ser uma ferramenta útil quer para o Colégio de Neurocirurgia da Ordem dos Médicos, os diretores do internato médico, os orientadores de formação, a Sociedade Portuguesa de Neurocirurgia e os diretores de serviço hospitalares, permitindo identificar aspetos com potencial de melhoria em Portugal.

\section{Objetivos finais}

Este estudo pretende caracterizar a formação especializada no internato médico de Neurocirurgia em Portugal, assim como a satisfação dos atuais internos ou recém-especialistas, em 2019, no que diz respeito à formação teórica, prática e à sua avaliação. Num segundo momento, este estudo pretende também determinar uma possível associação entre a satisfação e os fatores estudados.

\section{MATERIAL E MÉTODOS}

Estudo quantitativo, observacional e transversal baseado num questionário original enviado eletronicamente aos internos e recém-especialistas de Neurocirurgia entre outubro e dezembro de 2019.

\section{Questionário}

O questionário utilizado foi elaborado em língua portuguesa, constituído por questões com resposta única e múltipla (Apêndice 1: https://www.actamedicaportuguesa.com/ revista/index.php/amp/article/view/15110/Apendice_01. pdf). Trata-se de um trabalho original, tendo algumas perguntas sido adaptadas do mencionado artigo de MN Stienen, publicado na Acta Neurochirurgica com a autorização da EANS. ${ }^{9}$

O conjunto de perguntas encontra-se dividido nas categorias como 'Formação teórica', 'Formação prática', 'Avaliação do internato', 'Números cirúrgicos' e 'Questões (gerais) finais'. As perguntas de escolha múltipla que caracterizam a satisfação dos médicos internos seguiram uma escala de Likert de cinco pontos variando de 'Muito satisfeito' a 'Muito insatisfeito!' e uma opção 'Não existe / Não se adapta'.

O questionário foi colocado na plataforma Google Forms e o link para o mesmo divulgado através de correio eletrónico. O seu preenchimento foi voluntário e anónimo, tendo ficado disponível online por sete semanas, entre 21 de outubro e 9 de dezembro de 2019.

\section{Aprovação ética}

Para este tipo de estudo, o consentimento foi considerado tacitamente dado no preenchimento do questionário, pois a página de rosto contém informações sobre o estudo e o consentimento informado.

O protocolo de pesquisa foi submetido e aprovado pelo Comité de Ética do Centro Hospitalar de Lisboa Ocidental (CHLO), após parecer favorável e autorização do Conselho de Administração.

\section{Análise estatística}

Realizou-se análise descritiva através do cálculo de medidas de localização central (média/mediana) e de dispersão (desvio-padrão/amplitude interquartil) para as variáveis quantitativas contínuas, conforme apropriado. Para as variáveis qualitativas, foram calculadas frequências absoluta e relativa (percentagem). Para estudar a associação entre diversas variáveis foi utilizado o teste de qui-quadrado (ou o teste de Fisher, quando as frequências esperadas fossem inferiores a 5) para variáveis categóricas, e testes $t$ de Student/ANOVA ou Mann-Whitney-U/Kruskal Wallis para variáveis contínuas, conforme apropriado. O nível de significância estatística utilizado foi de $5 \%(p=0,05)$. O pacote estatístico utilizado foi o IBM SPSS Statistics versão 21.

Em alguns casos, para facilitar a análise e maior compreensão dos resultados, a escala de cinco categorias foi dicotomizada para satisfeito/satisfied, que inclui de 'Muito satisfeito' (Very satisfied), 'Satisfeito' (Satisfied) e 'Indiferente' (Indifferent); e não satisfeito/non-satisfied, que inclui 'Insatisfeito' (Dissatisfied), 'Muito insatisfeito' (Very dissatisfied) e 'Não existe / Não se adapta' (Does not exist / does not adapt).

\section{RESULTADOS \\ Caracterização básica}

Houve um total de 37 respostas e todas foram incluídos no estudo. A Tabela 1 mostra a caracterização básica dos participantes, incluindo parâmetros demográficos. A maioria das respostas vieram de internos do primeiro, terceiro e quinto ano. De modo a proteger o anonimato, foi decidido apenas identificar a origem dos internos por dois grupos Norte e Centro/Sul/Ilhas.

Segundo a Tabela 1, a maioria dos centros de formação tende a ter cerca de sete internos e 13 especialistas, havendo um número muito reduzido de especialistas doutorados.

\section{Formação teórica: caracterização}

A Tabela 2 mostra a existência (ou não) e o tipo de reuniões multidisciplinares revelada pelos participantes. A reunião de tumores primários e secundários do sistema nervoso central (SNC) parece ser a mais frequentemente encontrada $(86,1 \%)$ nos centros portugueses, seguida da de epilepsia (58,3\%), estimulação cerebral profunda (DBS) $(44,4 \%)$ e Neurovascular $(38,9 \%)$.

\section{Formação teórica: satisfação}

A Fig. 1 mostra a distribuição da satisfação com sessões clínicas (incluindo journal clubs), reuniões multidisciplinares, reuniões de morbimortalidade, sessões de Anatomia e acesso a um Laboratório de Anatomia, 
Tabela 1 - Caracterização da amostra

\begin{tabular}{|c|c|c|}
\hline Variável & & $\begin{array}{c}\text { Resultados } \\
(n=37)\end{array}$ \\
\hline Idade (anos) & Mediana (IQR) & $29( \pm 4,0)$ \\
\hline Sexo masculino & n (\%) & $29(78,4)$ \\
\hline \multicolumn{3}{|l|}{ Região } \\
\hline Norte & $\mathrm{n}(\%)$ & $17(45,9)$ \\
\hline Centro, Sul e Ilhas & $\mathrm{n}(\%)$ & $20(54,1)$ \\
\hline \multicolumn{3}{|l|}{ Ano de Internato } \\
\hline $1 .^{\circ}$ ano & n (\%) & $8(21,6)$ \\
\hline $2 .^{\circ}$ ano & $\mathrm{n}(\%)$ & $4(10,8)$ \\
\hline $3 .^{\circ}$ ano & $\mathrm{n}(\%)$ & $7(18,9)$ \\
\hline $4 .^{\circ}$ ano & $\mathrm{n}(\%)$ & $4(10,8)$ \\
\hline $5 .^{\circ}$ ano & $\mathrm{n}(\%)$ & $8(21,6)$ \\
\hline $6 .^{\circ}$ ano & n (\%) & $4(10,8)$ \\
\hline Recém-especialista & $\mathrm{n}(\%)$ & $2(5,4)$ \\
\hline \multicolumn{3}{|l|}{ Caracterização dos serviços } \\
\hline N. ${ }^{\circ}$ de Internos & Mediana (IQR) & $7( \pm 3,0)$ \\
\hline N. ${ }^{\circ}$ de Especialistas & Média (DP) & $13( \pm 4,0)$ \\
\hline $\mathrm{N} .^{\circ}$ de Especialistas com doutoramento & Mediana (IQR) & $1( \pm 3,0)$ \\
\hline \multicolumn{3}{|l|}{ Neurocirurgia como primeira escolha } \\
\hline Sim & n (\%) & $32(86,5)$ \\
\hline \multicolumn{3}{|l|}{ Carga horária semanal referida } \\
\hline Total & Mediana (IQR) & $70( \pm 20,0)$ \\
\hline Bloco & Mediana (IQR) & $20( \pm 9,0)$ \\
\hline Enfermaria & Mediana (IQR) & $15( \pm 14,0)$ \\
\hline Urgência & Mediana (IQR) & $24( \pm 4,0)$ \\
\hline Consulta & Mediana (IQR) & $5( \pm 2,0)$ \\
\hline Estudo & Mediana (IQR) & $4( \pm 6,0)$ \\
\hline
\end{tabular}

Tabela 2 - Caracterização da existência de reuniões multidisciplinares segundo a amostra

\begin{tabular}{lcc}
\hline Reuniões multidisciplinares & & $\begin{array}{c}\text { Resultados } \\
(\mathrm{n}=37)\end{array}$ \\
\hline Epilepsia & $\mathrm{n}(\%)$ & $21(58,3)$ \\
DBS & $\mathrm{n}(\%)$ & $16(44,4)$ \\
Dor crónica & $\mathrm{n}(\%)$ & $5(13,9)$ \\
Lesões selares e paraselares & $\mathrm{n}(\%)$ & $18(50,0)$ \\
Tumores primários e/ou secundários do SNC & $\mathrm{n}(\%)$ & $31(86,1)$ \\
Coluna & $\mathrm{n}(\%)$ & $8(22,2)$ \\
Patologia neurovascular & $\mathrm{n}(\%)$ & $14(38,9)$ \\
Otoneurocirurgia ou base do crânio & $\mathrm{n}(\%)$ & $0(0)$ \\
Neurociências & $\mathrm{n}(\%)$ & $27(75,0)$ \\
\hline
\end{tabular}

contribuição para a formação médica pós-graduada, possibilidade de investigação clínica e saídas do serviço para cursos e/ou rotações (estágios). De uma maneira geral, os participantes não ficaram satisfeitos com as reuniões de morbimortalidade $(59,5 \%)$, a existência (ou melhor, a inexistência) de sessões / laboratório anatómico (89,2\%), a contribuição para a formação médica pós-graduada $(64,9 \%)$ nem com a possibilidade de fazer investigação clí- nica $(64,9 \%)$.

\section{Formação teórica: análise estatística extra e breve dis- cussão}

O grau de satisfação geral com as reuniões multidisciplinares parece estar relacionado com a frequência com que ocorrem nos centros portugueses e, portanto, particularmente com a de Epilepsia ( $p=0,001$ teste não 
paramétrico), DBS ( $p=0,006$ teste não paramétrico) e tumores do SNC ( $p=0,062$ teste não paramétrico).

A taxa de satisfação (cuja escala de cinco categorias

A

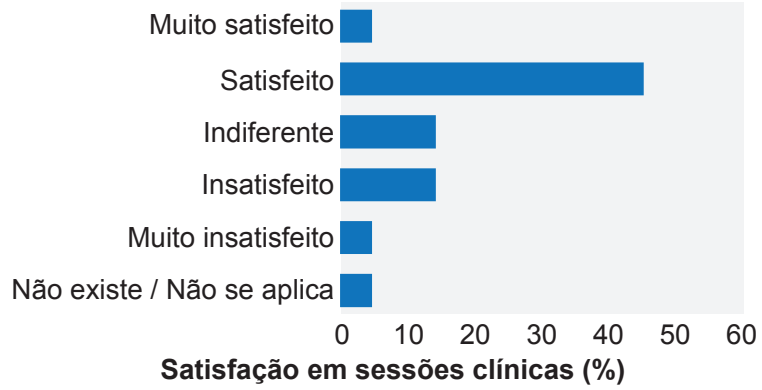

C

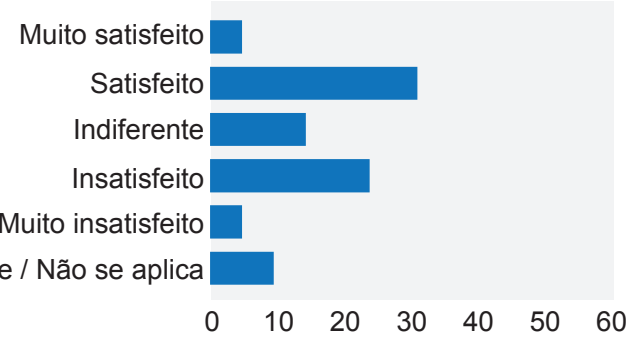

Satisfação em reuniões de morbimortalidade (\%)

E

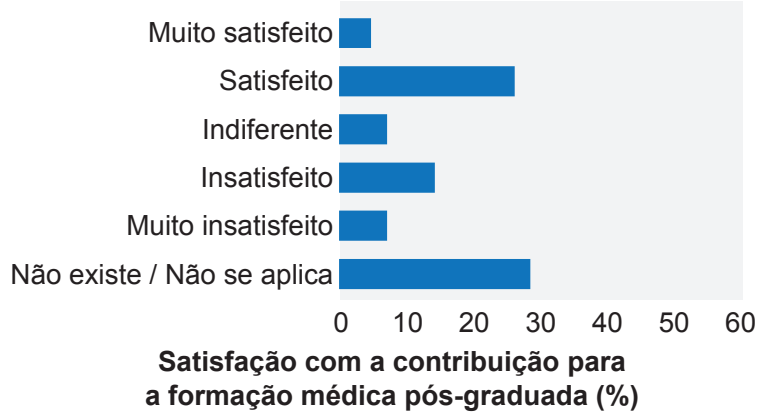

G

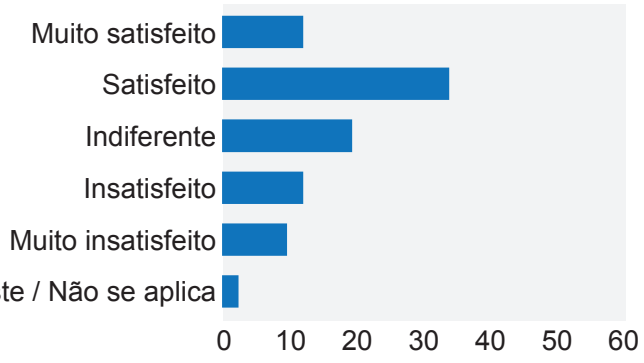

Satisfação com as saídas do serviço para cursos (\%) foi dicotomizada para satisfeito/satisfied e não satisfeito/ non-satisfied) com as reuniões de morbimortalidade parece estar associada ao ano de internato $(p=0,0036$ teste

B

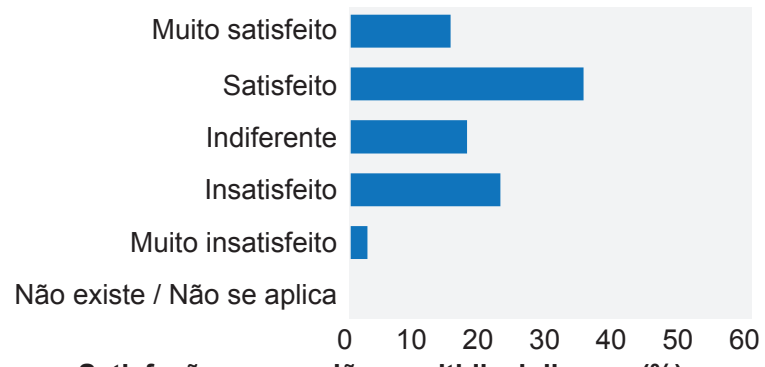

(D)

Satisfação em reuniões multidisciplinares (\%)

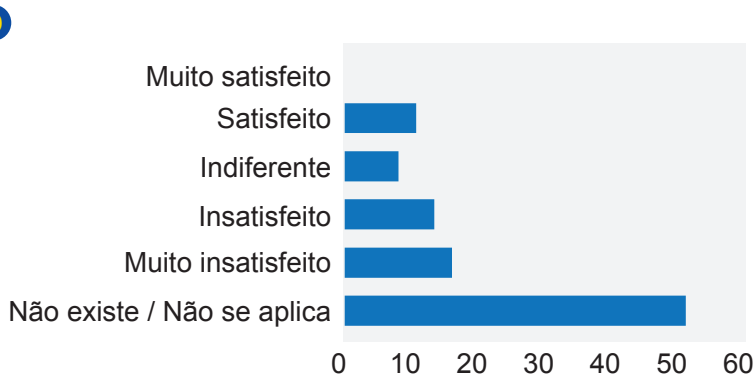

Satisfação em sessões anatómicas e acesso a um laboratório de anatomia (\%)

$\boldsymbol{P}$

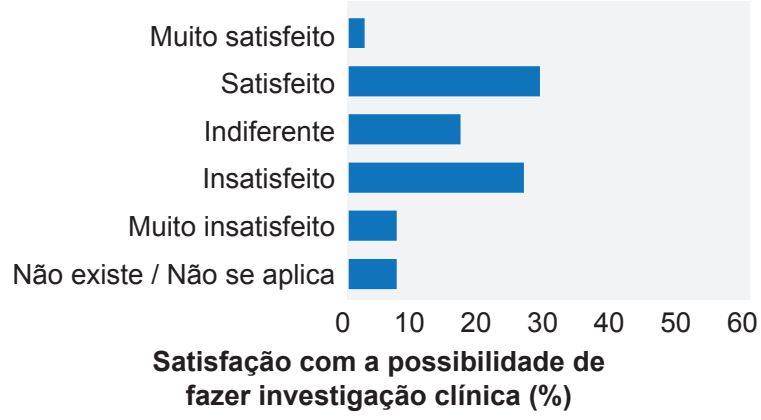

$\boldsymbol{\Theta}$

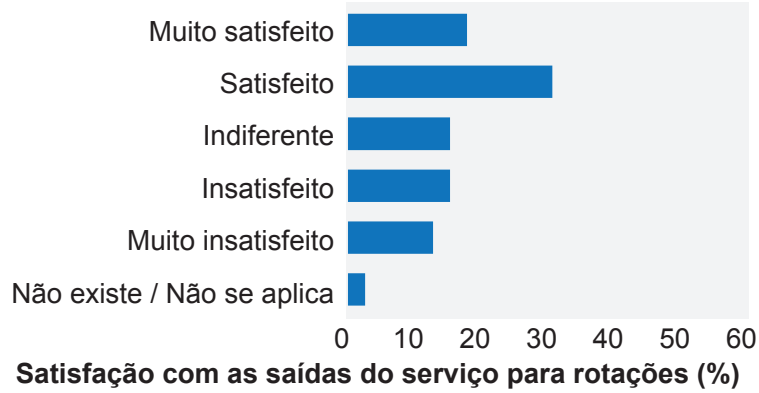

Figura 1 - Histograma do nível de satisfação com sessões clínicas (incluindo journal clubs) (A), reuniões multidisciplinares (B), reuniões de morbimortalidade (C), sessões anatómicas e acesso a um laboratório de Anatomia (D), contribuição para a formação médica pós-graduada $(E)$, possibilidade de fazer investigação clínica $(F)$ e saídas do serviço para cursos $(G)$ e rotações $(H)$. No eixo x foram colocadas as percentagens relativamente a graus de satisfação e no y os graus em escala de Likert de cinco pontos variando de "Muito satisfeito", "Satisfeito", "Indiferente", "Insatisfeito" e "Muito insatisfeito" e uma opção "Não existe / Não se adapta". 


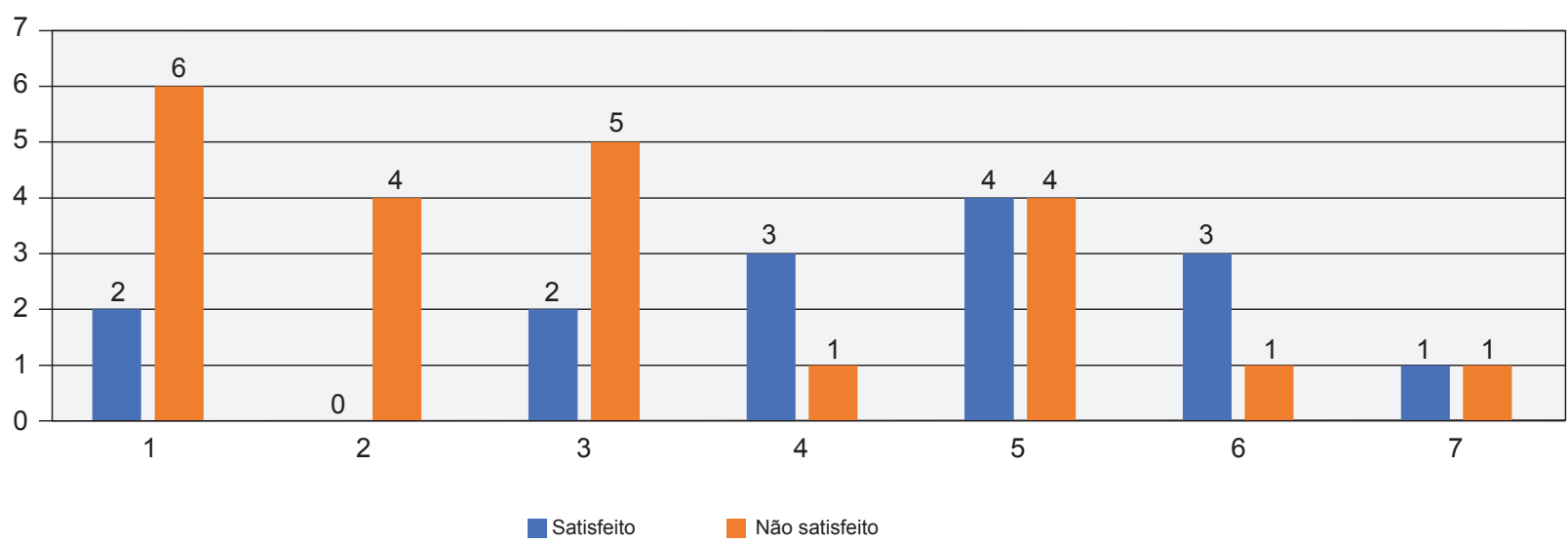

Figura 2 - Satisfação com as reuniões de Morbimortalidade em relação ao ano de internato. No eixo x apresenta-se o ano de internato, sendo o 7 correspondente ao ser recém-especialista. Cada ano mostra o número de internos satisfeitos e não satisfeitos.

não paramétrico) (Fig. 2), o que significa que os internos mais velhos parecem apreciar melhor este tipo de sessões. Além disso, o número de especialistas ( $p=0,017$ teste não paramétrico) e o número de médicos com doutoramento ( $p=0,009$ teste não paramétrico) mostraram também uma associação positiva com esta satisfação.

O maior grau de insatisfação expresso em relação às reuniões de morbimortalidade $(59,5 \%$ vs $40,5 \%)$, parece estar associado à sua inexistência em alguns centros. Relativamente à sua periodicidade, observa-se uma proporção contrária, com maior satisfação para reuniões com a periodicidade semanal $(70 \%$ de satisfação) e trimestral ( $71,4 \%$ de satisfação).

A satisfação com a possibilidade de fazer investigação parece estar associada ao número de horas dispensado (e provavelmente possível) por cada interno para estudo $(p=$ 0,008 teste não paramétrico).

O número de horas de permanência no serviço de Urgência, isto é, a realização de maior número de urgências, parece ter uma associação inversa na satisfação face à possibilidade de realização de cursos ( $p=0,049$ teste não paramétrico) e rotações ( $p=0,036$ teste não paramétrico).

\section{Formação prática: caracterização}

A caracterização da prática clínica está descrita na Tabela 1. Quanto à carga semanal do interno, esta corresponde a cerca de 70 horas e inclui 24 horas de urgência (não descriminando se juntas ou separadas em dois blocos de 12 horas), e horas no bloco operatório (BO) (que constitui, naturalmente, a maior incidência de horas, à exceção das realizadas em urgência).

\section{Formação prática: satisfação}

Em relação à formação prática, parece haver maior insatisfação apenas em relação às consultas $(56,8 \%)$ (ver Tabela 3).

\section{Formação prática: análise estatística extra e breve dis- cussão}

Foi realizada análise estatística (com testes não paramétricos) para verificar a associação entre a satisfação e eventuais influenciadores (variáveis contínuas), tais como número de turnos de urgência e de horas de urgência, para a secção da satisfação geral no SU; número de visitas à enfermaria, número de doentes observados/dia, camas, número de horas na enfermaria, número de internos e especialistas (que contem para a distribuição dos doentes), para a secção de enfermaria; número de períodos de consulta, número de doentes observados e número de horas em consulta, para a secção de consulta; número de horas no BO (em cirurgia) e número de internos e especialistas (que operem), para a satisfação geral da secção do BO bem como para a satisfação na participação no BO e ainda para a autonomia progressiva neste.

No caso da consulta, a satisfação geral (ou melhor, a insatisfação) como escala Likert inicial (não dicotomizada), parece estar associada à satisfação quanto ao número de períodos de consultas $(p=0,002)$, número de doentes observados $(p=0,016)$ e número de horas de consulta $(p=$ $0,008)$.

\section{Dados cirúrgicos: caracterização}

A Tabela 4 mostra o momento aproximado do internato (em meses) em que cada interno realizou a primeira cirurgia, por tipo de procedimento cirúrgico.

Tabela 3 - Caracterização da satisfação em relação às componentes da prática clínica

$\begin{array}{lcc} & \text { Satisfeito } & \text { Não-satisfeito } \\ \mathrm{n}(\%)\end{array}$


Tabela 4 - Momento da realização de primeira cirurgia, por procedimento cirúrgico, ao longo da formação especializada do internato médico

\begin{tabular}{lcccc}
\hline Variável & Medida de dispersão & $\begin{array}{c}\text { Resultado } \\
\text { (mês do internato) }\end{array}$ & $\begin{array}{c}\text { Intervalo } \\
\text { (min. - max.) } \\
\text { (mês do internato) }\end{array}$ & $\begin{array}{c}\text { Respostas } \\
\text { consideradas } \\
\mathrm{n}(\%)\end{array}$ \\
\hline Geral & Mediana (IQR) & $1,00( \pm 1,00)$ & $0-6$ & $36(97,3 \%)$ \\
Coluna lombar & Média (DP) & $11,41( \pm 1,50)$ & $2-24$ & $32(86,5 \%)$ \\
Coluna cervical & Mediana (IQR) & $13,00( \pm 13,00)$ & $2-30$ & $29(78,4 \%)$ \\
Patologia de LCR & Média (DP) & $5,95( \pm 4,30)$ & $0-16$ & $33(89,2 \%)$ \\
Craniotomia & Média (DP) & $6,59( \pm 3,88)$ & $0-12$ & $35(94,6 \%)$ \\
Traumatismo craniano & Média (DP) & $5,09( \pm 4,59)$ & $0-16$ & $33(89,2 \%)$ \\
Patologia oncológica craniana & Média (DP) & $16,70( \pm 8,15)$ & $3-36$ & $29(89,2 \%)$ \\
Nervos periféricos & Mediana (IQR) & $6,00( \pm 7,00)$ & $1-24$ & $34(91,9 \%)$ \\
Pediátrica & Média (DP) & $19,36( \pm 20,00)$ & $3-48$ & $24(64,9 \%)$ \\
\hline
\end{tabular}

Há uma tendência para que a primeira intervenção cirúrgica seja realizada no primeiro mês de internato e, no primeiro ano, em ordem crescente, a primeira cirurgia trauma craniano $(5,09 \pm 4,59$ meses $)$, posteriormente a de patologia de líquor (5,95 $\pm 4,3$ meses), nervos periféricos (6 \pm 7 meses), craniotomia $(6,59 \pm 3,88)$ e patologia lombar $(11,41 \pm 1,5$ meses). A cirurgia pediátrica é o último grupo de patologias a ser iniciada $(19,36 \pm 20$ meses $)$ e a que apresenta maior inconsistência.

É interessante realçar que existe um intervalo importante de respostas na nossa amostra, e que encontrámos diferenças estatisticamente significativas quando comparámos as respostas de internos dos centros do Norte versus Centro / Sul / Ilhas, em relação à primeira cirurgia lombar $(p=0,047)$, craniotomia $(p=0,020)$, e especialmente para oncologia (tumores cerebrais) ( $p=0,007)$ e cirurgia de nervos periféricos $(p=0,001)$, como as demais com o teste $U$ de Mann-Whitney), parecendo sugerir que os internos dos centros do Norte iniciam esse tipo de cirurgias mais tarde (Tabela 5). Numa tentativa de identificar que outras variáveis contribuíram para o timing da primeira cirurgia, o ano de internato atual (primeiro ao terceiro ano versus quarto, até ao especialista recente) parece ter alguma importância, já que se identificou, por exemplo, que as cirurgias de ner- vos periféricos se têm vindo a iniciar mais tarde nos anos recentes ( $p=0,049$, teste $U$ de Mann-Whitney), enquanto que o género do interno, na nossa amostra, não demonstrou ter qualquer impacto.

\section{Dados cirúrgicos: satisfação e análise estatística extra}

Em relação à satisfação com a participação no $\mathrm{BO}$, esta parece estar bastante relacionada com os tempos da primeira cirurgia da patologia das vias de líquor ou líquido cefalorraquidiano (LCR) ( $p=0,017$ Teste de Kruskal-Wallis) e da cirurgia de trauma encefálico ( $p=0,011$ segundo o teste de Kruskal-Wallis). Para a autonomia progressiva no $\mathrm{BO}$, parece que existe associação apenas com a patologia do LCR, entendendo-se que os que iniciam este tipo de cirurgias mais tarde são os mais insatisfeitos ( $p=0,027$ segundo o teste de Kruskal-Wallis).

\section{Avaliação}

Parece haver satisfação geral com o exame anual $(59,5 \%)$, mas não com o final $(37,8 \%)$. Os resultados posteriores podem sofrer um viés pelo fato de a maioria dos participantes ainda não ter passado por esta avaliação final.

De acordo com as diretrizes nacionais, o exame anual

Tabela 5 - Momento da realização de primeira cirurgia, por procedimento cirúrgico, ao longo da formação especializada do internato médico, no Norte versus Centro / Sul / llhas.

\begin{tabular}{lcccc}
\hline Variável & $\begin{array}{c}\text { Medida de } \\
\text { dispersão }\end{array}$ & $\begin{array}{c}\text { Resultado } \\
\text { (mês do internato) } \\
\text { Norte }\end{array}$ & $\begin{array}{c}\text { Medida de } \\
\text { dispersão }\end{array}$ & $\begin{array}{c}\text { Resultado } \\
\text { (mês do internato) } \\
\text { Centro / Sul / Ilhas }\end{array}$ \\
\hline Geral & Mediana (IQR) & $1,00( \pm 0,0)$ & Média (DP) & $1,5( \pm 1,1)$ \\
Coluna lombar & Mediana (IQR) & $12,50( \pm 4,0)$ & Mediana (IQR) & $7,0( \pm 15,0)$ \\
Coluna cervical & Média (DP) & $16,75( \pm 5,8)$ & Mediana (IQR) & $11,0( \pm 22,0)$ \\
Patologia de LCR & Média (DP) & $3,84( \pm 4,7)$ & Média (DP) & $4,9( \pm 3,8)$ \\
Craniotomia & Mediana (IQR) & $8,00( \pm 7,0)$ & Média (DP) & $4,9( \pm 6,3)$ \\
Traumatismo craniano & Média (DP) & $5,58( \pm 5,2)$ & Média (DP) & $4,5( \pm 3,9)$ \\
Patologia oncológica craniana & Mediana (IQR) & $18,00( \pm 8,0)$ & Média (DP) & $12,2( \pm 7,3)$ \\
Nervos periféricos & Média (DP) & $10,66( \pm 7,0)$ & Média (DP) & $4,0( \pm 1,8)$ \\
Pediátrica & Mediana (IQR) & $22,00( \pm 17,0)$ & Média (DP) & $17,2( \pm 15,2)$ \\
\hline
\end{tabular}


deve ser dividido em três partes. Segundo as respostas do questionário, este parece conter parte teórica em 89,2\% das instituições, parte prática em $83,8 \%$ e avaliação curricular em $94,6 \%$ dos casos. É de notar novo viés aqui, pelo facto de haver alguns internos de primeiro ano que poderão ter respondido sem ainda ter passado por este exame.

\section{Outras questões}

O trabalho em equipa com rotação $(59,5 \%)$ ao longo do internato parece ser o modo de trabalho/organização mais comum nos centros de formação, com satisfações nas várias componentes práticas. As restantes modalidades presentes são numa equipa fixa ao longo do internato $(13,5 \%)$, apenas/sobretudo com o orientador de formação $(2,7 \%)$ e nenhuma das anteriores $(24,3 \%)$.

A Tabela 6 mostra as subespecialidades marcadamente presentes nos centros da nossa amostra. Quando questionados sobre a satisfação quanto à possibilidade em se dedicar a uma determinada subespecialidade neurocirúrgica, $48,6 \%$ dos internos estão satisfeitos, porém 51,4\% não (com um número importante de casos indiferentes ou não existentes / não se aplica).

Em $81,1 \%$ dos casos, o questionando afirmou deliberadamente que gostaria de ser contratado como Especialista no mesmo hospital onde está a ser - ou foi - treinado.

\section{DISCUSSÃO}

Em geral, o nosso estudo aponta para uma certa tendência de insatisfação quanto à formação teórica, mas de satisfação quanto à formação prática (principalmente cirúrgica) dos internos portugueses.

Quanto à formação teórica, a insatisfação incide especialmente nas sessões anatómicas/acesso a laboratório anatómico $(89,2 \%)$, que não existem na maioria dos centros de formação, e na participação na formação médica $(64,9 \%)$ e em investigação clínica (64,9\%), já que, apesar da maior dos centros de formação se situarem em hospitais universitários, ligados a uma faculdade de medicina, não existe propriamente um estágio ou rotação neurocirúrgica na maioria das nossas faculdades de medicina portuguesas. Além disso, o recurso a internos para assegurar as urgências dificulta

Tabela 6 - Caracterização das áreas específicas existentes nos serviços da nossa amostra

\begin{tabular}{lll}
\hline Áreas específicas & & $\begin{array}{c}\text { Resultados } \\
(\mathrm{n}=37)\end{array}$ \\
\hline Coluna & $\mathrm{n}(\%)$ & $31(83,8)$ \\
Patologia neurovascular & $\mathrm{n}(\%)$ & $34(91,9)$ \\
Oncologia & $\mathrm{n}(\%)$ & $29(78,4)$ \\
Trauma craniano & $\mathrm{n}(\%)$ & $28(75,7)$ \\
DBS & $\mathrm{n}(\%)$ & $25(67,6)$ \\
Dor & $\mathrm{n}(\%)$ & $20(54,1)$ \\
Epilepsia & $\mathrm{n}(\%)$ & $23(62,2)$ \\
Lesões selares & $\mathrm{n}(\%)$ & $31(83,8)$ \\
Base do crânio & $\mathrm{n}(\%)$ & $26(70,3)$ \\
Pediatria & $\mathrm{n}(\%)$ & $24(64,9)$ \\
\hline
\end{tabular}

muito a possibilidade de realização de mais investigação científica, de deslocações quer a nível nacional quer a nível internacional para um curso ou estágio, oportunidades educativas extremamente importantes e gratificantes para quase todos internos. Mesmo assim existe, pelo menos durante o último ano de internato, o hábito de permitir que o interno se concentre numa área/subespecialidade, neurocirúrgica de preferência, ou que em que identifica menor conhecimento, dentro ou fora do seu Serviço. Portugal parece estar bastante atrasado relativamente a outros países no cenário da investigação e publicação científica, provavelmente em resultado de menor tradição e de falta de condições essenciais como recursos financeiros, infraestruturas, tempo e disponibilidade para tal. Contudo, esta questão não foi claramente identificada, nomeadamente a relação com publicações e estudos a decorrer em cada centro, dado a especificidade e a extensão já longa do questionário.

A nossa amostra corrobora a média de mais de 60 horas de trabalho demonstrada por Stienen em 2016, o que nos coloca no topo dos países europeus com mais horas de trabalho. ${ }^{15}$

Quanto à formação prática, parece existir maior insatisfação apenas em relação às consultas (56,8\%), que se encontra aparentemente associado ao descontentamento face ao número continuamente crescente de doentes encaminhados para consulta neurocirúrgica - número de períodos de consulta $(p=0,002)$, número de pacientes ( $p=$ $0,016)$ e número de horas em consultas $(p=0,008)$, que ultrapassam sempre a capacidade de resposta dos hospitais.

Estes resultados estão de acordo com o inquérito da EANS que demonstrou que os internos portugueses estão entre os mais satisfeitos no que diz respeito à organização dos journal clubs, ao início da prática cirúrgica e ao contacto com um grande número de patologias.

Apesar da intenção inicial ser avaliar números cirúrgicos propriamente ditos, percebeu-se nos questionários-teste que estes tipos de questões levariam mais tempo a ser respondidas, o que prejudicaria a adesão ao questionário, já em si bastante longo. Assim, decidiu-se avaliar a atividade cirúrgica com base no momento (timing) da primeira cirurgia. Os resultados parecem bastante justos e correspondentes ao esperado, até o facto da primeira cirurgia em neurocirurgia pediátrica ser mais tardia, pela sua especificidade, e bastante variável, provavelmente por não ser uma subespecialidade presente em todos os centros, o que obriga muitas vezes os internos a fazerem uma rotação fora. Além disso, não existem muitos casos pediátricos hoje em dia, pelo que a sua maioria são encaminhados para os centros de referência, para que estes também não percam habilidade ou conhecimento sobre o assunto. É interessante perceber que existe ainda um importante intervalo no que diz respeito aos momentos da primeira cirurgia na nossa amostra: quando comparamos os centros do Norte versus os do Centro / Sul / Ilhas, verifica-se que os internos dos centros do Norte tendem a iniciar mais tarde determinado tipo de cirurgias. A pergunta que devemos fazer é se 
o problema reside em políticas e/ou tradições dos diferentes serviços, na disponibilidade de patologias/casos ou na necessidade de um número mínimo de cirurgias mais adequado.

\section{Limitações}

Este estudo apresenta várias limitações.

Apesar da reduzida amostra deste estudo, se pensarmos que anualmente existem cerca de 50 internos e eventualmente 9 a 11 recém-especialistas, 37 respostas correspondem a cerca de $61 \%$ a $63 \%$ de colaboração desta população-alvo, o que não parece ser um fraco resultado para um questionário longo e potencialmente controverso.

Por se tratar da primeira vez que se elabora e realiza este tipo de inquérito no nosso país, os autores preferiram basear-se noutro já publicado, ${ }^{9}$ adaptando-o à realidade portuguesa. Porém, de alguma forma, chegou-se a um ponto em que parecia haver dois tipos de estudo num só, ou dois tipos de perguntas e objetivos distintos - por um lado, caracterizar centros de formação e, por outro, analisar a satisfação com a formação neurocirúrgica - o que levou a um maior número de questões e necessidade de tempo para respondê-las. Esta situação poderá ter comprometido a participação da população-alvo, à partida com pouco tempo livre. A formulação do questionário e o reduzido tamanho da amostra tornaram quase impossivel encontrar as associações que se desejavam, o que dificultou bastante a análise estatística. $\mathrm{O}$ trabalho apresenta vários vieses, tais como o facto de poder não ter chegado informaticamente a todos os elementos da população-alvo, poder ser sujeito a várias respostas por um mesmo indivíduo, incluir perguntas que poderão não se adaptar tão bem a todos os elementos da população-alvo (como as questões relativas aos tempos cirúrgicos e avaliações, entre outras) e poder sofrer de 'recall bias', como por exemplo os internos mais novos poderem saber com maior exatidão determinados dados, nomeadamente quanto a timings cirúrgicos, dado estarem mais próximos desse momento. Apesar de sabermos que a qualidade da educação não está necessariamente relacionada com a satisfação, acreditamos que seja diretamente proporcional. Os autores reconhecem que a realização de estudos de qualidade passará primeiramente por definir critérios oficiais que ultrapassam a sua competência.

Ainda assim, consideramos ser útil apresentar este estudo, uma vez que poderá constituir um ponto de partida para estudos mais direcionados para determinadas questões, como por exemplo a identificação de associações entre o momento da primeira cirurgia e os números finais da cirurgia, o papel do tutor no internato, etc. e sobretudo, um alerta para a situação atual da formação em Neurocirurgia em Portugal, suspeitando-se que o crescente aumento do número de internos e recém-especialistas nos vários centros possa vir eventualmente a retirar currículo cirúrgico aos internos mais novos.

Em última instância, os autores esperam que este estudo possa servir como ponto de partida para outros estudos mais focados em determinadas áreas da formação, melhorias intra e inter-hospitalares e eventualmente para a elaboração do novo programa de formação do internato médico de Neurocirurgia.

\section{CONCLUSÃO}

De um modo geral, os internos portugueses parecem estar satisfeitos com a maioria dos sectores de formação propostos (algumas exceções estão relacionadas sobretudo com a indisponibilidade de alguma formação teórica e excesso de consultas).

Este estudo cumpriu o objetivo principal de ser um ponto de partida para a caracterização dos centros neurocirúrgicos portugueses e da satisfação no internato de formação especializada em Neurocirurgia. Esperamos que possa contribuir para a melhoria e uniformização do ensino da Neurocirurgia em Portugal.

\section{AGRADECIMENTOS}

Os autores agradecem à SPNC (Sociedade Portuguesa de Neurocirurgia) e ao Colégio de Especialidade de Neurocirurgia da Ordem dos Médicos por permitirem a distribuição deste inquérito e a realização deste estudo. Agradece-se também a importante contribuição estatística do Professor Paulo Nogueira, integrante do Departamento de Investigação do Centro Hospitalar de Lisboa Ocidental.

\section{CONTRIBUTO DOS AUTORES}

LND, PPL: Elaboração do estudo e redação do artigo. CR: Conceção do trabalho, revisão crítica do estudo.

JC: Revisão crítica do estudo.

\section{PROTEÇÃO DE PESSOAS E ANIMAIS}

Os autores declaram que os procedimentos seguidos estavam de acordo com os regulamentos estabelecidos pelos responsáveis da Comissão de Investigação Clínica e Ética e de acordo com a Declaração de Helsínquia da Associação Médica Mundial atualizada em 2013.

\section{CONFIDENCIALIDADE DOS DADOS}

Os autores declaram ter seguido os protocolos do seu centro de trabalho acerca da publicação de dados.

\section{CONFLITO DE INTERESSES}

Os autores declaram não haver conflito de interesses.

\section{FONTES DE FINANCIAMENTO}

Os autores não receberam financiamento para este estudo.

Disponível em: https://www.sns.gov.pt/wp-content/uploads/2017/09/ RRH-Neurocirurgia-Aprovada-a-6-setembro-2017.pdf.

\section{REFERÊNCIAS}

1. Vaz R, Natário A, Vara Luiz C, Carvalho E, Oliveira F, Maia G, et al. Rede de referenciação hospitalar de Neurocirurgia. [consultado 2019 abr 26] 
2. Ryu W, Chan S, Sutherland G. Supplementary educational models in Canadian Neurosurgery residency programs. J Can Sci Neurol. 2017;44:177-83.

3. Stienen M, Gempt J, Gautschi O, Demetriades A, Netuka D, Kuhlen D, et al. Neurosurgical resident training in Germany. J Neurol Surg Part Cent Eur Neurosurg. 2017;78:337-43.

4. Tso M, Dakson A, Ahmed SU, Bigder M, Elliott C, Guha D, et al. Operative landscape at Canadian Neurosurgery residency programs. Can J Neurol Sci. 2017;44:415-9.

5. Xu T, Evins Al, Lin N, Chang J, Hu G, Hou L, et al. Neurosurgical postgraduate training in China: moving toward a national training standard. World Neurosurg. 2016;96:410-6.

6. Portugal. Portaria n. ${ }^{\circ}$ 146/1998. Diário da República, I-B Série, n. ${ }^{\circ} 57$ (1998/03/09). p.920-33.

7. Ordemdosmedicos. Inquérito de Idoneidade e Capacidade Formativa. [consultado 2020 mar 16]. Disponível em: https://ordemdosmedicos.pt/ inquerito-de-idoneidade-e-capacidade-formativa-26/.

8. Union Européene des Médecins Spécialistes (UEMS) - Section of Neurosurgery. European training requirements for the specialty of neurosurgery. European standards of postgraduate medical specialist training in neurosurgery. [consultado 2019 abr 28] Disponível em: https://www.uems.eu/_data/assets/pdf_file/0007/44449/UEMS2015.34-European-Training-Requirements-in-Neurosurgery.pdf.

9. Stienen M, Netuka D, Demetriades A, Ringel F, Gautschi O, Gempt J, et al. Neurosurgical resident education in Europe - results of a multinational survey. Acta Neurochir. 2016;158:3-15.

10. Bina R, Lemole $G$, Dumont $T$. On resident duty hour restrictions and neurosurgical training: review of the literature. $J$ Neurosurg. 2016;24:842-8.

11. Jean W, Felbaum D. Impact of training and practice environment on academic productivity of early career academic neurosurgeons. World Neurosurg. 2019;121:e892-7.

12. Karsy M, Henderson F, Tenny S, Guan J, Amps J, Friedman A, et al. Attitudes and opinions of US neurosurgical residents toward research and scholarship: a national survey. J Neurosurg. 2019;131:252-63.

13. Khan N, Saad H, Oravec C, Norrdahl S, Fraser B, Wallace D, et al An snalysis of publication productivity during residency for 1506 neurosurgical residents and 117 residency departments in North America. Neurosurgery. 2019;84:857-67.

14. Ottenhausen M, Anetsberger S, Kleffmann J, Schuss P, Konczalla $\mathrm{J}$, Krawagna $\mathrm{M}$, et al. Risk factors for dropping out of neurosurgical residency programs - a survey study. World Neurosurg. 2018;120:e100 6.16 .

15. Spetzler R. Progress of women in neurosurgery. Asian J Neurosurg 2011;6:6.

16. Stienen M, Netuka D, Demetriades A, Ringel F, Gautschi O, Gempt J, et al. Working time of neurosurgical residents in Europe-results of a multinational survey. Acta Neurochir. 2016;158:17-25.

17. Wolfe S, West J, Hunt M, Murad G, Fox W, Dow J, et al. A comparison of the existing wellness programs in neurosurgery and institution champion's perspectives. Neurosurgery. 2019;84:1149-55.

18. Woodrow S, Gilmer-Hill H, Rutka J. The neurosurgical workforce in North America: a critical review of gender issues. Neurosurgery. 2006;59:749 58. 